\title{
The choice of analysis of variance models in repeated measurements analysis-on the effect of glaucoma surgery on retinal structures
}

\author{
Kevin Gillmann $\mathbb{1}^{1} \cdot$ Harsha L. Rao $\mathbb{1}^{2} \cdot$ Kaweh Mansouri ${ }^{1,3}$ \\ Received: 4 October 2019 / Accepted: 11 October 2019 / Published online: 13 November 2019 \\ (c) The Royal College of Ophthalmologists 2019
}

\section{To the Editor:}

We always welcome comments and contributions to research, and as such, we read with great interest the remark by Ebrahimi et al. on the statistical methods of our recent publication entitled "Effect of surgical intraocular pressure lowering on retinal structures-nerve fibre layer, foveal avascular zone, peripapillary and macular vessel density: 1year results". In their comment, the authors suggested that repeated measure analysis of variance (ANOVA) was better suited for the evaluation of repeated measurements in a set cohort than one-way ANOVA. Indeed, in the aforementioned article, we analysed the variations in intraocular pressure, retinal nerve fibre layer thickness, foveal avascular zone parameters and vascular densities of several retinal and peri-papillary sub-regions in 40 eyes at several time-points, before and after filtering surgery. As Ebrahimi et al. rightly pointed out, this constitutes a dependent comparison of repeated measurements, for which we fully agree that repeated measure ANOVA is the appropriate statistical test and, in fact, is precisely the test that was used in our analysis.

Indeed, contrary to what Ebrahimi et al. implied, we did not specify the type of ANOVA model used in our article's methods section. Therefore, we can only speculate that our omission led the comment's authors to wrongly speculate that our analysis was based on one-way ANOVA. For this reason, we would like to take the opportunity of this response to add this short complement to the methods section of our original article, confirming the use of repeated measures ANOVA in our analysis.

\section{Compliance with ethical standards}

Conflict of interest The authors declare that they have no conflict of interest.

Publisher's note Springer Nature remains neutral with regard to jurisdictional claims in published maps and institutional affiliations.
Kaweh Mansouri

kwmansouri@gmail.com

1 Glaucoma Research Center, Montchoisi Clinic, Swiss Visio Network, Lausanne, Switzerland

2 Narayana Nethralaya, Bangalore, India

3 Department of Ophthalmology, University of Colorado School of Medicine, Denver, CO, USA 